\title{
Análisis Numérico Unidimensional del Proceso de Infiltración en Suelos Parcialmente Saturados Reforzados con Geosintéticos Permeables
}

\author{
Numerical Analysis of the One-dimensional Infiltration Process in Unsaturated \\ Soils Reinforced with Permeable Geosynthetics
}

\author{
J. C. Guzmán-Martínez iD, E. F. García-Aristizábal iD, A. N. Gallego-Hernández iD, C. A. \\ Vega-Posada
}

\begin{abstract}
This article presents a numerical analysis of the water infiltration process on one-dimensional unsaturated soil and soilgeosynthetic columns. The analysis focuses on evaluating the presence of the capillarity barrier under different scenarios of rainfall intensity, initial suction and soil permeability. The results showed a low capacity of the geosynthetic to work as a drainage material when the initial suction in the soil is high, allowing the accumulation of water in the soil above the geosynthetic layer. This research aims to contribute to the state of knowledge on the hydraulic response of geosynthetic-reinforced soils.
\end{abstract}

Index Terms - One-dimensional numerical analysis, Capillary barrier, Permeable geosynthetic, Unsaturated soils.

Resumen- Este estudio presenta un análisis numérico del proceso de infiltración de agua en columnas unidimensionales de suelo y suelo-geosintético bajo condiciones parcialmente saturadas. El análisis se enfoca en evaluar la presencia de la barrera capilar bajo distintos escenarios de lluvia, presión de poros negativa inicial y permeabilidad del suelo. Los resultados mostraron una baja capacidad del geosintético para actuar como material filtrante cuando el suelo se encuentra en bajos niveles de presión de poros, permitiendo la acumulación de agua en el suelo ubicado por encima del geosintético. Esta investigación aporta al conocimiento de la respuesta hidráulica en la interacción suelogeosintético.

Palabras claves - Análisis numérico unidimensional, Barrera capilar, Geosintético permeable, Suelo parcialmente saturado.

Este manuscrito fue enviado el 07 de mayo de 2018 y aceptado el 20 de marzo de 2019.

Los autores agradecen a la Universidad de Antioquia, a su grupo de Investigación en Infraestructura GII y a su semillero de Geología y Suelos, donde se desarrolló este trabajo

J.C. Guzmán-Martínez, Candidato a Maestría, Escuela Ambiental, Facultad de Ingeniería, Universidad de Antioquia, Colombia. Cll 70\#52-21. A.A.1226 (juanc.guzman@udea.edu.co)

E.F. García-Aristizábal, Profesor Asociado, Escuela Ambiental, Universidad de Antioquia, Colombia. Cll 70\#52-21. A.A.1226 (edwin.garcia@udea.edu.co)

\section{INTRODUCCIÓN}

T A infiltración de la lluvia representa el principal factor detonante en las fallas de llenos, terraplenes y suelos compactados [1], [2]. En la búsqueda de resolver dicha problemática, los geosintéticos han surgido como materiales sustitutos a los materiales granulares convencionales, los cuales tienen como finalidad facilitar la disipación del exceso de presión de poros generada por eventos repetitivos de infiltración [3]. Durante varios años, se han registrado numerosos casos de éxito con el uso de estos materiales geosintéticos. Chattopadhyay \& Chakravarty [4] resaltan la efectividad del geosintético dentro de estructuras de suelo, con el propósito de desarrollar una rápida consolidación. Tan et al. [5] mediante ensayos de laboratorio de suelo residual junto a capas de geosintético permeable, demostraron la eficacia del geosintético no tejido en reducir el exceso de presión de poros utilizando la teoría de consolidación de Terzaghi [6], la cual se desarrolló en condiciones de saturación total en el suelo.

Sin embargo, en la mayoría de casos, los geosintéticos son dispuestos en la masa de suelo por encima del nivel freático, en donde los poros contienen agua y aire [7], induciendo presiones de poros negativas (succión) durante y después de la construcción. Durante este periodo las estructuras de suelo reforzadas están expuestas a factores como la evapotranspiración y la lluvia de infiltración, generando variaciones en el contenido de agua existente, razón por la cual la condición de saturación parcial predomina en el suelo superficial [8].

Varios estudios han reportado el efecto de los geosintéticos no tejidos como material de refuerzo en estructuras de suelo

A.N. Gallego-Hernández, Profesora Asistente, Escuela Ambiental, Universidad de Antioquia, Colombia. Cll 70\#52-21. A.A.1226 (alba.gallego@udea.edu.co)

C.A. Vega-Posada, Profesor Asociado, Escuela Ambiental, Universidad de Antioquia, Colombia. Cll 70\#52-21. A.A.1226 (carlosa.vega@udea.edu.co) 
bajo condiciones parcialmente saturadas. Clough \& French [9] fueron los primeros en presentar el comportamiento del geosintético no tejido como drenaje dentro de una estructura de pavimento. Este estudio reportó el aumento en el contenido de agua en las capas de suelo por encima del geotextil, Richardson [10] informó que los geosintéticos no tejidos empleados como capas drenantes en taludes no permitían el flujo de agua de forma fácil y rápida en los suelos por encima de la capa del geosintético, concluyendo que la falla de un talud estudiado se generó a partir de la condición impuesta por el geosintético (citado en [8,p.288]).

Resultados similares a los anteriores se han reportado por varios investigadores, reflejando incertidumbres y deficiencias en el comportamiento de los geosintéticos no tejidos bajo dicha condición. Iryo \& Rowe [3] evaluaron de forma numérica el efecto del geosintético no tejido en terraplenes sujetos a lluvia, mediante el uso de elementos finitos, encontrando que el geosintético puede retardar el flujo de agua bajo el escenario de presión de poros negativas (succión); no obstante, puede actuar como un material drenante en presiones de poros positivas. García et al. [11] reportó experimentalmente la respuesta hidráulica y mecánica de los geosintéticos permeables en un modelo a escala de terraplén sujeto a lluvia de infiltración. El resultado muestra que el suelo por encima de la capa de geosintético aumenta su contenido de agua considerablemente con respecto al suelo por debajo del geosintético, generando fallas locales observadas en el proceso de infiltración debido a la acumulación de agua por encima de las capas del geosintético. Bathurst et al. [12] midió la respuesta de la presión de agua utilizando ensayos de infiltración en columnas de arena con y sin geosintético, detectando que el geotextil actuó como una barrera capilar hasta alcanzar succiones cercanas a cero en la interface con el geosintético.

Recientemente, Thuo et al. [13] empleó modelos numéricos en 2 dimensiones, con el uso de elementos finitos para simular el efecto de la barrera capilar, observando que para taludes no saturados reforzados con geotextil no tejido, éstos actúan como una barrera de humedad en lugar de actuar como un material drenante, desarrollando presiones de poros positivas en el suelo inmediatamente por encima del geotextil. Jana et al. [14] modeló mediante elementos finitos un flujo transitorio similar al presentado por Thuo et al. [13], con el fin de modelar la respuesta de muros de suelo reforzado con geosintético no tejido con suelo fino sujeto a lluvia de infiltración. Ellos observaron que la conductividad hidráulica del geotextil se ve reducida dentro de altos niveles de succión, impidiendo el flujo de infiltración, y de esta manera reduciendo de esta manera la resistencia del suelo ubicado por encima del geotextil, resaltando que: "el uso de los geotextiles no tejidos requiere una investigación critica adicional" [p.11].

A pesar del avance investigativo mencionado anteriormente sobre el proceso de infiltración en sistemas suelo-geotextil, el uso de columnas como modelo de análisis de la barrera capilar ha sido poco empleado; además, la interacción hidráulica en la interface de estos materiales no ha sido abordada ampliamente bajo un análisis crítico de las variables que intervienen en el proceso. Es por esto que el presente estudio tiene como alcance estudiar el comportamiento hidráulico de sistemas de suelo con y sin geotextil mediante la variación de la presión de poros y el grado de saturación a partir del análisis numérico empleado en columnas unidimensionales utilizando un modelo acoplado viscoplástico de elementos finitos propuesto por Oka et al. [15].

Además, este trabajo pretende evaluar el efecto de la barrera capilar en el proceso de infiltración y realizar un análisis paramétrico que permita determinar la influencia de las condiciones iniciales y las características de la lluvia en la respuesta hidráulica de los materiales. Finalmente, el presente estudio pretende aportar en el estado del conocimiento sobre el comportamiento complejo de las estructuras reforzadas con geomateriales sujetos a infiltración de lluvia en condiciones parcialmente saturadas.

\section{MetodologiA}

\section{A. Modelo acoplado infiltración-deformación}

El modelo empleado es llamado COMVI (Consolidation Multiphase Viscoplastic Analysis) y fue desarrollado en la Universidad de Kyoto, Japón. Este simula el proceso de lluvia de infiltración en condiciones parcialmente saturadas basadas en la teoría de medios porosos (e.g. [16]). A continuación, se presentan las ecuaciones de dicha teoría y el modelo acoplado de infiltración-deformación utilizado para el análisis.

\section{B. Tensor de esfuerzos}

El tensor de esfuerzos se plantea mediante la formulación propuesta por Jommi [17], definen "skeleton stress" como la variable de esfuerzo en la relación constitutiva para la estructura del suelo, denominada el esfuerzo efectivo generalizado $\sigma_{i j}{ }^{\prime}$. El tensor de esfuerzos $\sigma_{i j}$ se obtiene como la suma de los esfuerzos parciales.

$\sum_{\alpha} \sigma_{i j}^{\alpha}=\sigma_{i j}(\alpha=\mathrm{S}, \mathrm{W}, \mathrm{G})$

$\mathrm{S}=$ Solido, $\mathrm{W}=$ Líquida, $\mathrm{G}=\mathrm{Gas}$

$\sigma_{i j}^{S}=\sigma_{i j}^{\prime}+n^{S} P^{F} \delta_{i j}$

$\sigma_{i j}^{W}=n^{W} P^{W} \delta_{i j}$

$\sigma_{i j}^{G}=n^{G} P^{G} \delta_{i j}$

Donde $P^{W}$ y $P^{G}$ es la presión de poros del agua y del aire, respectivamente, $n$ representa la porosidad, $n^{\alpha}$ es la fracción volumétrica de la fase $\alpha$ y $P^{F}$ es el promedio de las presiones según el grado de saturación $(s)$, calculado a partir de (5):

$P^{F}=\mathrm{s} P^{w}+(1-\mathrm{s}) P^{G}$

Mediante las Ecuaciones 1 a 5 se obtiene la formulación del esfuerzo efectivo generalizado (ver Ecuación 6), el cual es usado como el esfuerzo básico en el modelo de suelos parcialmente saturados.

$\sigma_{i j}^{\prime}=\sigma_{i j}-P^{F} \delta_{i j}$

\section{Conservación de masa}

La conservación de la masa viene dada por (7): 
$\frac{\partial}{\partial t}\left(n^{\propto} \rho_{\propto}\right)+\left(n^{\propto} \rho_{\propto} v_{i}^{\alpha}\right)$

$i=0 \quad \propto=S, W, G$

En la que $\rho_{\propto}$ es la densidad del material y $v_{i}^{\alpha}$ es la velocidad de la fase $\alpha$. Suponiendo que las derivadas espaciales de la fracción de volumen $n^{\propto}$ y las densidades de material $\rho_{\propto}$ son despreciables y suponiendo que las partículas del suelo y el agua son incompresibles, $\overline{\dot{\rho}}_{S}=0$ y $\bar{\rho}_{W}=0$, las leyes de conservación pueden expresarse como funciones de saturación de agua $s$ y porosidad $n$, es decir:

$-\dot{n}+(1-n) v_{i, i}^{S}=0$

$s D_{i i}+\dot{s} n=-V_{i . i}^{W}$

$(1-s) D_{i i}-\dot{s} n+(1-s) n \frac{\dot{\rho_{G}}}{\rho_{G}}=-V_{i, i}^{G}$

Donde $v_{i}^{S}$ es la velocidad de la fase sólida, $D_{i i}$ es el estiramiento volumétrico, y $V_{i}$ la velocidad aparente. En la ecuación (9), se supone que la densidad del agua es constante. Para describir los cambios en la densidad del aire, se usa la ecuación para el gas ideal.

\section{Conservación de momento}

El balance de momento para cada fase es dado por:

$n^{\propto} \rho_{\propto} \dot{v}_{i}^{\alpha}=\sigma_{j i, j}^{\alpha}+\rho_{\propto} n^{\propto} \bar{F}_{i}-\sum_{y} D^{\propto y}\left(v_{i}^{\alpha}-v_{i}^{y}\right)$

$\propto=S, W, G$

En la que $\bar{F}_{i}$ es la aceleración por gravedad o la fuerza de gravedad por unidad de masa y $D^{\propto y}\left(D^{\propto y}=D^{y \propto)}\right.$ son los parámetros que describen la interacción entre las fases $\propto$ y $y$, que se define como:

$D^{W S}=\frac{\left(n^{W}\right)^{2} \rho w g}{k^{W}}, \quad D^{G S}=\frac{\left(n^{G}\right)^{2} \rho G g}{k^{G}}$

En la que $k^{W}$ y $k^{G}$ son los coeficientes de permeabilidad para la fase acuosa y la fase aire, respectivamente, que se dan en $L / T$ (unidades). Dejando de lado la aceleración y la interacción entre el aire y el agua, la ecuación del equilibrio del momento se reduce a la ecuación de equilibrio para cada fase, obtenida como:

$\sigma_{j i, j}^{\prime}+\left(n^{S} P^{F}\right)_{i . j}+\rho_{S} n^{S} \bar{F} i-D^{S W}\left(v_{i}^{S} v_{i}^{W}\right)-D^{S G}\left(v_{i}^{S} v_{i}^{G}\right)=0$

$\left(n^{W} P^{W}\right)_{i, j}+\rho_{W} n^{W} \bar{F}_{i}-D^{W S}\left(v_{i}^{W} v_{i}^{S}\right)=0$

$\left(n^{G} P^{G}\right)_{i, j}+\rho_{G} n^{G} \bar{F}_{i}-D^{G S}\left(v_{i}^{G} v_{i}^{S}\right)=0$

Cuando asumimos que la derivada espacial de la fracción volumétrica $n_{, i}^{\alpha}$ es despreciable, las leyes de Darcy para las fases agua y aire se obtiene a partir de (14) y (15), respectivamente:
$V_{i}^{W}=n^{W}\left(v_{i}^{W}-v_{i}^{S}\right)=\frac{k^{W}}{W g}\left(P_{, i}^{W}+\rho_{W} \bar{F}_{i}\right)$

$V_{i}^{G}=n^{G}\left(v_{i}^{G}-v_{i}^{S}\right)=\frac{k^{G}}{\rho_{G} g}\left(P_{, i}^{G}+\rho_{G} \bar{F}_{i}\right)$

La suma de (13)-(17) conduce a:

$\sigma_{j i, j}+\rho \bar{F}_{i}=0$

\section{E. Curva de retención de agua}

La función que permite relacionar el contenido de humedad y la succión es la curva de retención de agua. En esta investigación se propone emplear la ecuación de Van Genuchten [18], al ser la uno de los modelos que mejor describe el comportamiento hidráulico [19].

$s=s_{\text {min }}+\left(s_{\text {máx }}-s_{\text {min }}\right)\left\{1+\left(\alpha P^{C}\right)^{n^{\prime}}\right\}^{-m}$

$m=1-\frac{1}{n^{\prime}}$

Donde las variables $\alpha, n^{\prime}$ y $m$ son parámetros de ajuste de la forma de la curva característica de retención de agua, teniendo que $s_{\text {máx }}$ y $s_{\min }$ son los valores máximos y mínimos para saturación, respectivamente. Los efectos del grado de saturación en la permeabilidad del aire y del agua se muestran a partir de las ecuaciones 21 y 22, respectivamente:

$k^{W}=k_{s}^{W} S^{a}\left\{1-\left(1-s^{\frac{1}{m}}\right)^{n^{\prime}}\right\}$

$k^{G}=k_{s}^{G}(1-s)^{b}\left\{1-\left(s^{\frac{1}{m}}\right)^{n^{\prime}}\right\}$

Donde $a$ y $b$ son parámetros propios del material, $k_{s}^{G}$ y $k_{s}^{W}$ son el coeficiente de permeabilidad para el aire bajo condiciones de suelo seco y el coeficiente de permeabilidad para el agua bajo condiciones de saturación total, respectivamente. Para más detalle sobre la formulación del modelo se recomienda al lector remitirse a [20].

\section{F. Análisis numérico}

Recientemente, las soluciones numéricas se han usado para analizar problemas en suelos parcialmente saturados, debido a la facilidad de establecer condiciones iniciales y de frontera que permiten modelar múltiples capas de suelo, diferentes intensidades de lluvias y diferentes parámetros hidráulicos [20]. Adicionalmente, el análisis de infiltración vertical unidimensional en suelos no saturados se convierte en un asunto de interés debido a la necesidad de comprender la respuesta altamente no lineal que exhiben estos suelos y su interacción con otros materiales.

Con la metodología de análisis que se presentará, se pretende estudiar la respuesta hidráulica que se da en la interacción del suelo con un geosintético. En la metodología empleada, las variables independientes son la presión de poros de agua $\left(P^{W}\right)$, la presión de poros del aire $\left(P^{G}\right)$ y la velocidad nodal $(u)$. En la configuración de los elementos finitos se contemplan elementos 
cuadriláteros de 8 nodos, los cuales son empleados para calcular desplazamientos mediante integración gaussiana. Cuatro de estos nodos se emplean para calcular presiones de poros de agua y aire en el centro de cada elemento. Aunque el modelo presentado permite el análisis de deformación unidimensional de los materiales estudiados, este estudio se concentra en analizar sólo la respuesta hidráulica en la interacción de estos materiales.

\section{G. Condiciones de frontera}

Las simulaciones se realizaron en sistemas de suelo con y sin geosintético. La geometría y las condiciones de frontera en la configuración suelo-geosintético se muestran en la Fig.1. Las alturas de los elementos que componen la columna varían según la proximidad que exista con la capa de geosintético, siendo los más cercanos de menor tamaño, con el propósito de generar una adecuada transición entre los materiales modelados a partir de elementos finitos.

La presión de poros inicial (succión) impuesta en todos los elementos es de $-50 \mathrm{kPa}$ y el nivel de agua se ubica por debajo de la columna, esto con el fin de representar una condición seca de los materiales inicialmente de manera uniforme. En el fondo de la columna se permite el paso de agua (condición drenada) y la presión de agua en los poros en estos nudos es igual a cero. Las paredes laterales se colocan como fronteras impermeables, la presión de aire inicial $P^{G}$ es asumida como cero, y la parte superior de la columna permite el ingreso de la lluvia durante 90 horas.

En los análisis presentados, la intensidad de lluvia es dada en relación a la permeabilidad saturada del suelo, empleándose una lluvia constante y menor que la permeabilidad saturada del suelo $\left(I / k_{s}^{w}<1.0\right)$. Adicionalmente, se estudió el efecto de las características hidráulicas del suelo y geosintético en el proceso de infiltración, por medio de un análisis paramétrico con diferentes valores de intensidad de lluvia, presión de poros negativa inicial y permeabilidad.

Los parámetros del suelo fueron los de una arcilla limosa empleada por Kimoto et al. [21] en simulaciones del modelo acoplado en Diques. En relación con los parámetros del geosintético se utilizaron los resultados de los ensayos realizados por Garcia et al. [11], excepto el valor de la permitividad, el cual corresponde a las propiedades de un geotextil no tejido de uso común en Colombia. Para este propósito se utilizó como fuente el catálogo de productos PAVCO (Geotextil NT 3000). Los parámetros del suelo y el geotextil se presentan en las Tablas I y II, respectivamente.

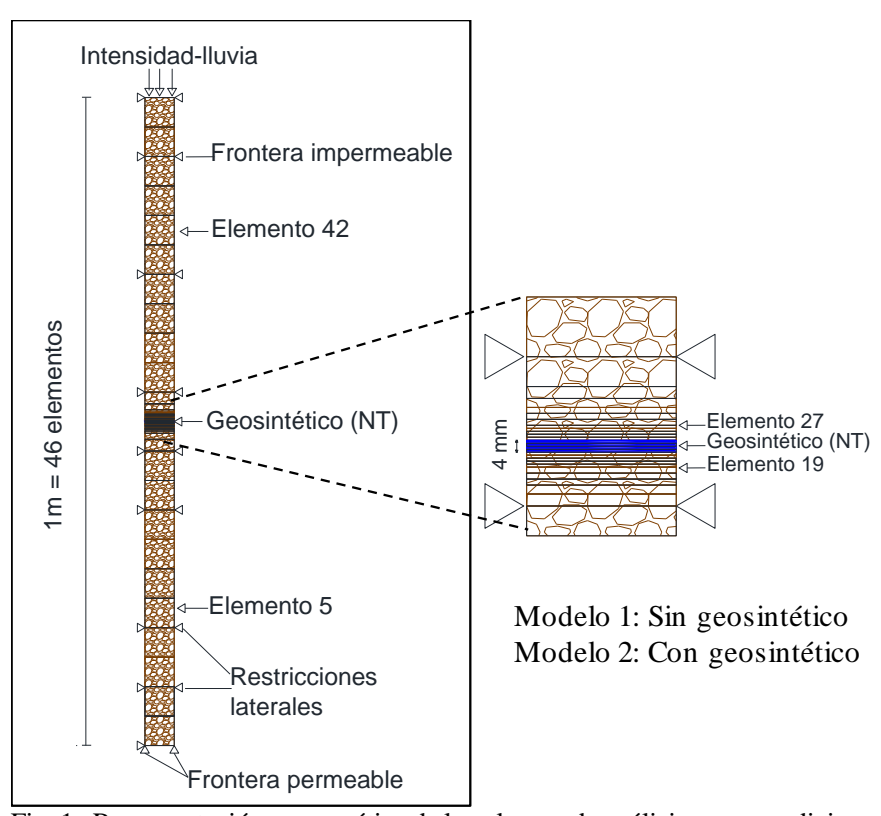

Fig. 1. Representación esquemática de la columna de análisis y sus condiciones de frontera.

TABLA I

PARÁMETROS BÁSicos DEL SUELO

\begin{tabular}{|c|c|c|}
\hline Simbolo & Parámetro & Valor \\
\hline$k_{s}^{W}$ & permeabilidad saturada del agua $(\mathrm{m} / \mathrm{s})$ & $10^{-6}$ \\
\hline$k_{s}^{G}$ & permeabilidad del gas en condiciones secas $(\mathrm{m} / \mathrm{s})$ & $10^{-3}$ \\
\hline$e_{0}$ & relación de vacíos inicial & 1.03 \\
\hline$S_{I}$ & parámetro de succión & 0.2 \\
\hline$S_{d}$ & parámetro de succión & 5.0 \\
\hline$S_{\max }$ & saturación máxima & 0.99 \\
\hline$S_{\min }$ & saturación minima & 0.0 \\
\hline$a$ & parámetro de coeficiente de permeabilidad del agua & 1.0 \\
\hline$b$ & parámetro de coeficiente de permeabilidad del agua & 1.0 \\
\hline & \multicolumn{2}{|l|}{$\begin{array}{c}\text { TABLA II } \\
\text { PARÁMETROS BÁSICOS DEL GEOSINTÉTICO } \\
\end{array}$} \\
\hline Simbolo & Parámetro & Valor \\
\hline$N T$ & Tipo de geosintético & No tejido \\
\hline$\mu_{\mathrm{A}}$ & Masa por unidad de área $\left(\mathrm{g} / \mathrm{m}^{2}\right)$ & 400 \\
\hline$t_{G T X}$ & Espesor $(\mathrm{mm})$ & 4 \\
\hline$n_{G T X}$ & Porosidad & 0.89 \\
\hline$e_{O G}$ & Relación de vacíos inicial & 8.10 \\
\hline$S_{\max }$ & saturación máxima & 0.88 \\
\hline$S_{\min }$ & saturación minima & 0.01 \\
\hline$k^{W}$ & Permitividad $\left(\mathrm{s}^{-1}\right)$ & \multirow{2}{*}{$\begin{array}{l}2 \\
8 \times 10^{-3}\end{array}$} \\
\hline$k_{s}^{W}$ & permeabilidad saturada del agua $(\mathrm{m} / \mathrm{s})$ & \\
\hline
\end{tabular}

Las curvas características de retención de agua para el suelo y el geosintético no tejido fueron determinadas a partir de la ecuación de Van Genuchten [18]. Para los parámetros $\alpha$ y $n^{\prime}$ correspondientes a la curva del suelo, se emplearon valores típicos de suelos como arena basados en Lu \& Likos [22], mientras que para el geosintético se ajustó la curva a partir de valores experimentales realizados en Garcia et al. [11]. En la Fig. 2 se presentan las curvas empleadas para ambos materiales. 


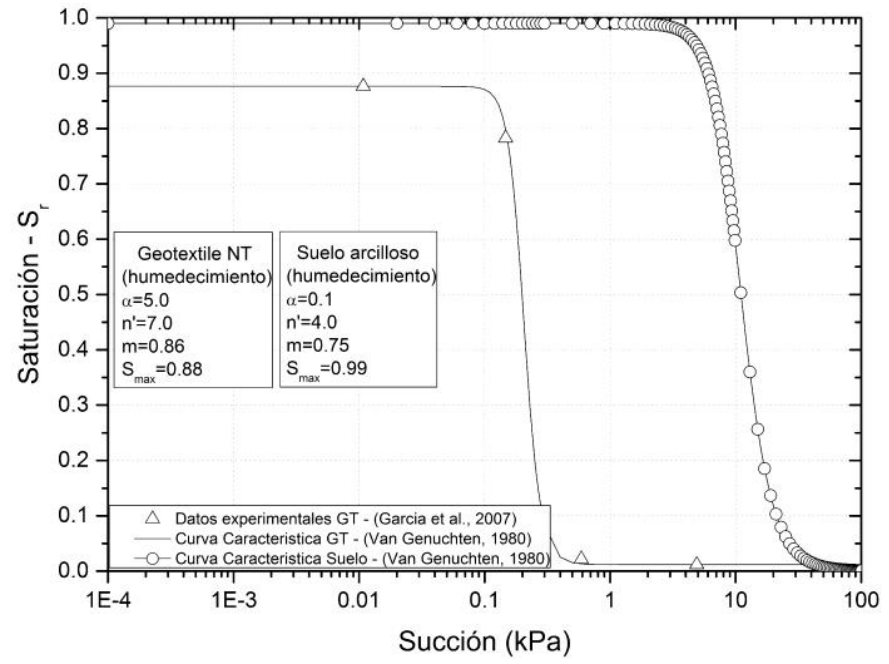

Fig. 2. Curvas características de retención para el suelo y el geotextil. Las curvas han sido ajustadas a la ecuación de Van Genuchten (1980).

\section{RESULTADOS}

\section{A. Resultados numéricos}

Las Fig. 3 y 4 presentan las variaciones temporales de la presión de agua en los poros y el grado de saturación respectivamente, para el Modelo 1 (Sin geosintético) durante el proceso de infiltración unidimensional. En la Fig. 3 es posible observar que, a medida que el tiempo de infiltración transcurre; el contenido de agua en el suelo es mayor, generando aumento de la presión de poros (disminución de la succión) a través de los elementos que componen la columna. Esta variación se manifiesta en primera instancia en los elementos superiores de la columna, debido a ser los primeros en interactuar con la lluvia. También se puede observar que los elementos ubicados en el fondo de la columna no presentan un aumento inmediato en el contenido de agua, reflejando la transición y flujo de agua a profundidad que ocurre entre los elementos, dicho comportamiento es similar al presentado por [12].

Posteriormente, en la hora 80 aproximadamente, se observa que los elementos presentan presiones de poros cercanas a los $10 \mathrm{kPa}$, mostrando que los elementos no se alcanzan a saturar completamente bajo las condiciones asumidas de lluvia. Esta respuesta es consistente con los resultados de la Fig. 4 donde se muestra la variación temporal de la saturación, en la cual ningún elemento alcanza un grado de saturación mayor a 0.7 del volumen de poros disponible en cada elemento, se infiere un proceso de infiltración continuo y permanente a través de todos los elementos de la columna.

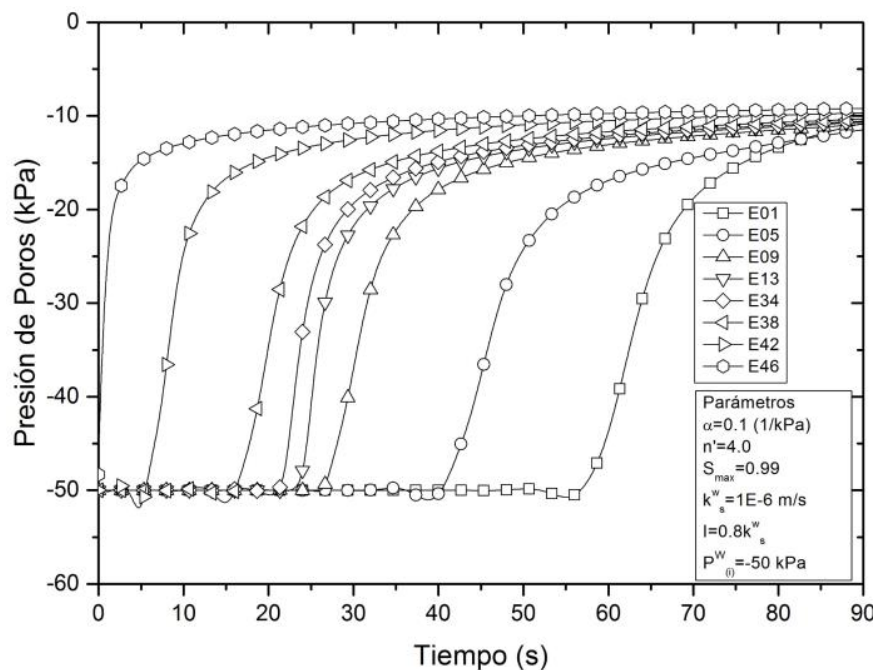

Fig. 3. Variación temporal de la presión de poros durante el proceso de infiltración.

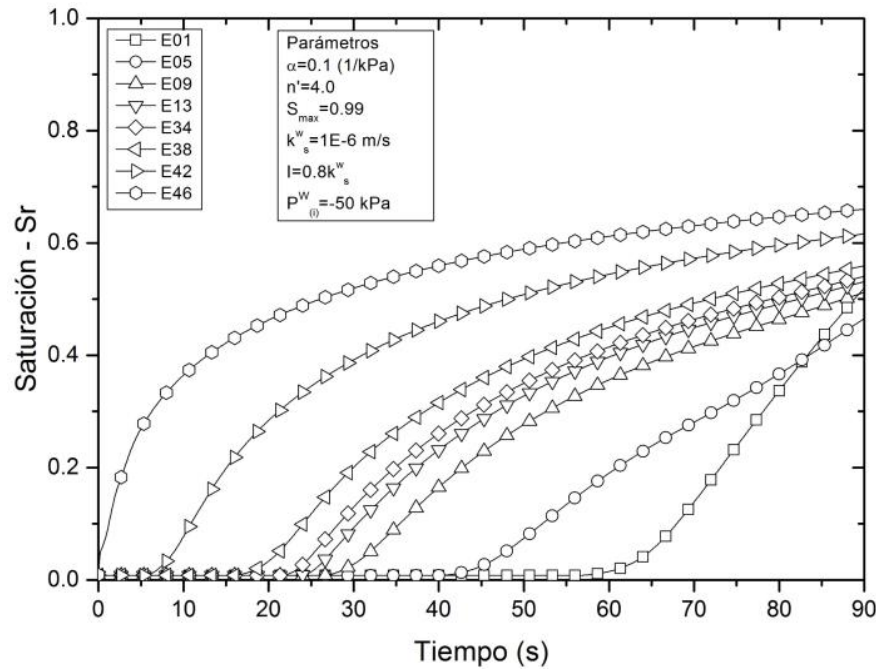

Fig. 4. Variación temporal de la saturación durante el proceso de infiltración.

En la Fig. 5 se muestra la comparación entre los dos modelos mediante el desarrollo de la presión de poros para los elementos 5 y 19 (bajo el geosintético) y 27 y 42 (sobre el geosintético), esto con el fin de evaluar el efecto impuesto por el geosintético. En el Modelo 2, se observa que los elementos con mayor presión de poros son los elementos 27 y 42 (E27G y E42G), los cuales están ubicados por encima del geosintético; no obstante, los elementos por debajo de este material (E05G y E19G) no presentan un incremento inmediato en la presión de poros; es decir, el agua no está drenando a través del geosintético.

Adicionalmente, la Fig. 5 muestra que el agua es retenida por encima del geosintético aumentando considerablemente la presión de poros por encima de este, y el agua solamente atraviesa el geosintético cuando se alcanzan presiones de poros cercanas a cero, por encima del geosintético. El paso del agua por el geotextil se da aproximadamente a las $75 \mathrm{~h}$ de infiltración. Este resultado es consecuente con el presentado experimentalmente en columnas de suelo reforzadas con diferentes geosintéticos permeables desarrolladas por Zornberg et al. [23]. De esta manera, el incremento de las presiones de poros en los elementos más cercanos por debajo del geosintético ocurre de manera súbita, como se puede evidenciar en el elemento 19 (E19G), el cual difiere del comportamiento 
de este mismo en el Modelo 1 (E19), donde se observa que el incremento en el contenido de agua se presenta de manera gradual a partir de la hora 22 aproximadamente.

En consecuencia, se observa que el proceso de infiltración en el Modelo 2 es interrumpido y discontinuo debido a la inclusión del geosintético como material de refuerzo en la columna, contrastando de manera notoria con los resultados del Modelo 1, el cual no incluye geosintético.

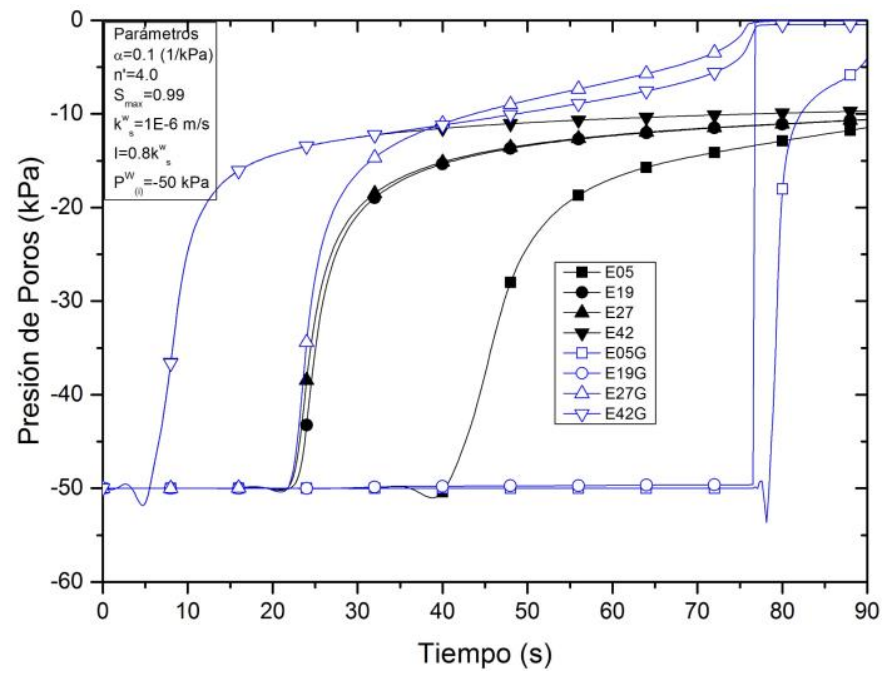

Fig. 5. Comparación entre el Modelo 1 y 2 en términos de la variación de la presión de poros.

El comportamiento mostrado anteriormente, también se puede explicar a partir de la Fig. 6, en la cual se muestra la variación espacial del grado de saturación a través de la columna para los tiempos de infiltración $\mathrm{t}=45$ y $80 \mathrm{~h}$, respectivamente, representando de manera más evidente la acumulación de agua en los elementos adyacentes por encima del geosintético.

La Fig. 6 muestra que en la hora 45 del proceso de infiltración, los contenidos de humedad a lo largo de todos los elementos del Modelo 1 han aumentado, siendo más altos en los elementos cercanos a la superficie de la columna y menores a medida que se aleja de la superficie. Por otro lado, en el caso del Modelo 2, se observa que las saturaciones son más altas en los elementos inmediatamente por encima del geotextil y muy bajas por debajo del mismo, indicando que el agua está siendo retenida por el geotextil. Los niveles de saturación de los elementos por encima del geotextil son mayores a los niveles de saturación de los elementos en la columna sin el geotextil.

Igualmente, la Fig. 6 muestra los perfiles de saturación presentados en ambos modelos a las 80 h de infiltración. Los resultados muestran que mientras que en el Modelo 1 los niveles de saturación están por debajo de 0.6 a través de toda la columna, en el Modelo 2 se observan saturaciones cercanas a 1.0 para todos los elementos por encima del geotextil. Esta respuesta indica que el agua se fue acumulando a través de este material debido a que el geotextil no facilita la filtración de agua a través de él. En condiciones parcialmente saturadas, el geosintético se convierte en un material que no contribuye efectivamente a la disipación de presiones de poros durante y después de la lluvia de infiltración, al inducir fronteras impermeables dentro de las estructuras de suelo, permitiendo la concentración de cuerpos de agua alrededor de él.

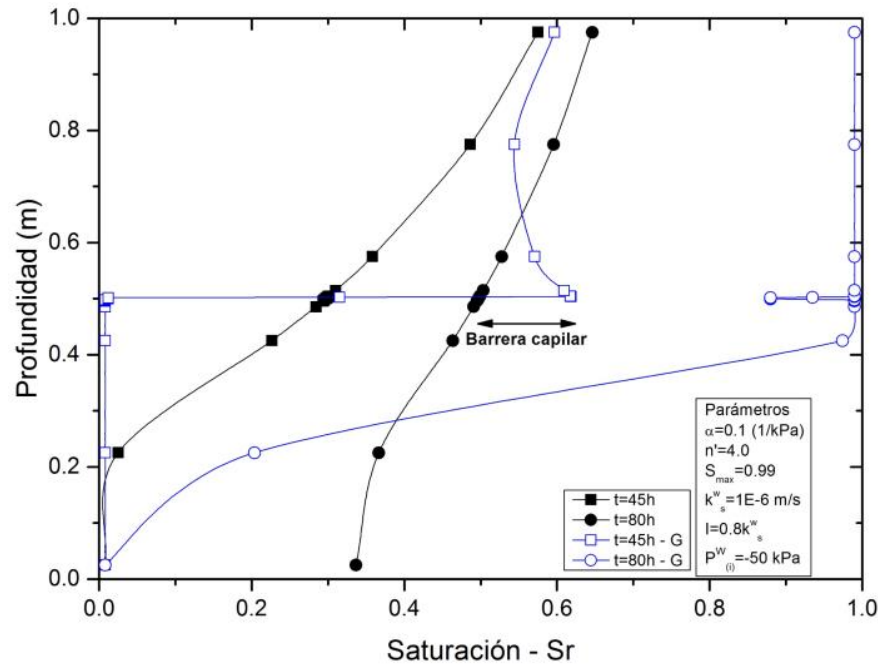

Fig. 6. Perfil de saturación para los Modelos 1 y 2 en los tiempos t=45 y 80 h.

Estos resultados son acordes a los previstos para cada material según su respectiva curva de retención de agua (SWCC, Fig. 2). Para el geosintético se observa que, en altos niveles de succión, el grado de saturación es muy bajo y solamente para succiones menores a $1 \mathrm{kPa}$ es cuando empieza a aumentar el contenido de agua en sus poros. También se observa el pequeño rango que presenta para pasar de bajos porcentajes de saturación a la saturación máxima $\left(S_{\max }=0.88\right)$ a partir de su elevada pendiente en la zona de transición, razón por la cual el cambio de la condición seca a saturada es casi de manera instantánea; por lo tanto, se infiere que el geosintético solo trabaja como material permeable en bajos rangos de succión, trayendo consigo el efecto de retención de agua cuando interactúa con suelos parcialmente saturados.

\section{B. Análisis paramétrico}

Con el fin de evaluar la influencia de la presión de poros negativa inicial del suelo, la intensidad de la lluvia y la permeabilidad saturada del suelo en el desarrollo de la barrera capilar, generada a partir de la interacción de dos materiales con distintas permeabilidades y sujetos a lluvia de infiltración, se realiza un análisis paramétrico con las siguientes variaciones:

\section{Presión de poros negativa inicial}

En la Fig. 7 se presentan las distribuciones de la presión de poros correspondiente a los elementos E19G (Bajo el geosintético) y E27G (sobre el geosintético) del Modelo 2 bajo distintos valores presión de poros inicial $(-100,-70,-50,-40$ y $30 \mathrm{kPa}$ ). De la Fig. 7, se puede observar que el incremento en la presión de poros (disminución de la succión) inicia en menor tiempo bajo el escenario de presión de poros inicial igual a $30 \mathrm{kPa}$, ocurriendo antes de la hora 20. Por otra parte, el elemento que tardó más tiempo para iniciar su proceso de acumulación de agua por encima del geosintético fue el de presión de poros igual a $-100 \mathrm{kPa}(\mathrm{E} 27 \mathrm{G})$, iniciando a las 28 horas aproximadamente. Por consiguiente, a medida que la presión de poros inicial establecida fue mayor $(-100 \mathrm{kPa}<-30$ $\mathrm{kPa}$ ) la concentración de agua en los elementos por encima del geomaterial se presentó de forma más rápida; en donde inicialmente el sistema suelo-geosintético con mayor succión 
(bajos niveles de presión de poros) otorga menos caminos disponibles para el flujo a través del geomaterial [8].

Así mismo, se analiza el comportamiento del elemento E19G, en la Fig. 7 se observa que la barrera capilar se supera después de la hora 75 para todas las presiones de poros iniciales dadas, donde se presenta un incremento drástico de la saturación, lo que implica que el agua sobre el geosintético ha pasado a través del material de refuerzo incrementando la presión de poros del suelo bajo este. Sin embargo, cabe destacar que para la presión de poros de $-30 \mathrm{kPa}$ dicha frontera impermeable permaneció por más tiempo y solo después de aproximadamente 80 horas de acumulación aproximadamente se permite el flujo por el geosintético. Adicionalmente, se observa una tendencia del tiempo requerido para sobrepasar dicha barrera, observando que para una presión de poros inicial de $-100 \mathrm{kPa}$; el proceso de saturación se da en 48 horas (entre los tiempos 28 y 76 horas) y para una presión de poros inicial de $-30 \mathrm{kPa}$ el proceso de saturación se da en 62 horas (entre las 18 y 80 horas). Esta respuesta muestra que, a menor presión de poros inicial, se presenta mayor facilidad para romper la barrera capilar en términos de tiempo.

Al resultado anterior se plantean varias alternativas de análisis. Una de estas alternativas parte de la premisa de que la barrera capilar genera un incremento en la capacidad de almacenamiento de agua en los suelos, en comparación a condiciones normales de drenaje [24], por lo tanto, cuando la acumulación de agua se da a bajos niveles de presión de poros la capacidad de retener agua es mayor, generando que el proceso de saturación y posteriormente alcanzar la presión necesaria para romper la barrera capilar se presente de forma más rápida, en comparación con los resultados del modelo sujeto a mayor presión de poros (-30 kPa). Otra de las hipótesis; es considerar el aumento en la capacidad de absorber agua por parte del suelo por debajo del geosintético bajo menores presiones de poros, que probablemente permita atraer de forma más rápida las partículas de agua retenidas en la parte superior del geotextil.

No obstante, la validez de este resultado debe ser corroborado por medio de estudios experimentales que argumenten dicha condición a partir del comportamiento físico, también se puede emplear la función de conductividad hidráulica de los materiales a interactuar (suelo-geosintético) para predecir el valor presión de poros en donde la ruptura de la barrera capilar se haga efectiva [23] y de esta manera poder evaluar el rendimiento de la barrera capilar bajo distintos escenarios de succión mediante la curva de retención y los parámetros de ajuste del modelo de Van Genuchten [18].

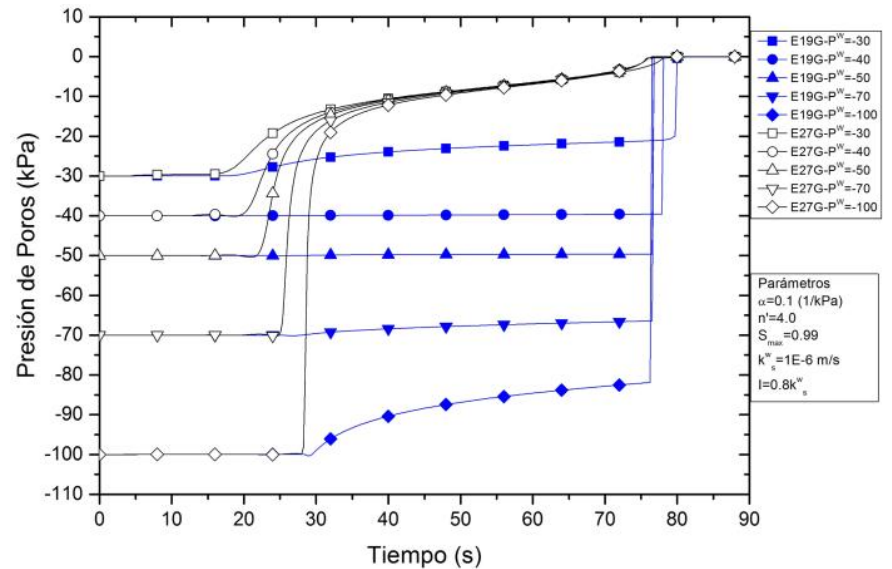

Fig. 7. Presión de poros de los elementos 27 y 19 del modelo 2 bajo distintos valores de presión de poros inicial (-30, -40, -50, -70 y -100 $\mathrm{kPa}$ ).

\section{Intensidad de lluvia}

En términos de evaluar la influencia de la intensidad en la interacción hidráulica del suelo-geosintético, se realizaron 4 simulaciones con diferentes intensidades menores o iguales a la permeabilidad saturada del suelo. Las intensidades estudiadas fueron $I=0.4 k^{W}, I=0.6 k^{W}, I=0.8 k^{W}$ y $I=k^{W}$. La Fig. 8 presenta el perfil de saturación para el Modelo 2 bajo las distintas intensidades establecidas previamente y para el tiempo de 45 horas de infiltración, tiempo en el cual no se ha superado la barrera capilar. La Fig. 8 muestra un grado de saturación casi nulo de los elementos por debajo del geosintético. En segunda instancia se puede observar conforme al incremento de la intensidad, que los elementos por encima del geosintético presentan mayor contenido de agua, siendo el de mayor grado de saturación el escenario con $I=k^{W}$.

En contraste con lo ocurrido en la hora 45, en el tiempo 80 se alcanza a romper la barrera capilar durante las mayores intensidades $\left(I=0.8 k^{W}\right.$ y $\left.I=k^{W} S\right)$, tal como se muestra en la Fig. 9. Para estas intensidades los elementos inferiores presentan un incremento en el grado de saturación. De este modo para el escenario propuesto de intensidad de lluvia igual a la permeabilidad saturada $\left(I=k^{W}\right)$; el sistema suelo-geosintético presenta una condición de presiones de poros cercanas a cero como efecto de superar el "tapón" generado por el geosintético y la mayor cantidad de agua que permite la acumulación de agua en los elementos superiores. Sin embargo, para los casos de $I=0.4 k^{W}$ y $I=0.6 k^{W}$ no se vence la barrera capilar, debido a que los elementos por encima del geotextil no han reducido la succión matricial en los niveles necesarios (cercanos a cero) para que el geosintético facilite el drenaje, presentando unas saturaciones aproximadas de 0.9 y 0.6 para $I=0.6 k^{W}$ y $I=0.4 k^{W}$, respectivamente, en el elemento cercano al geosintético. 


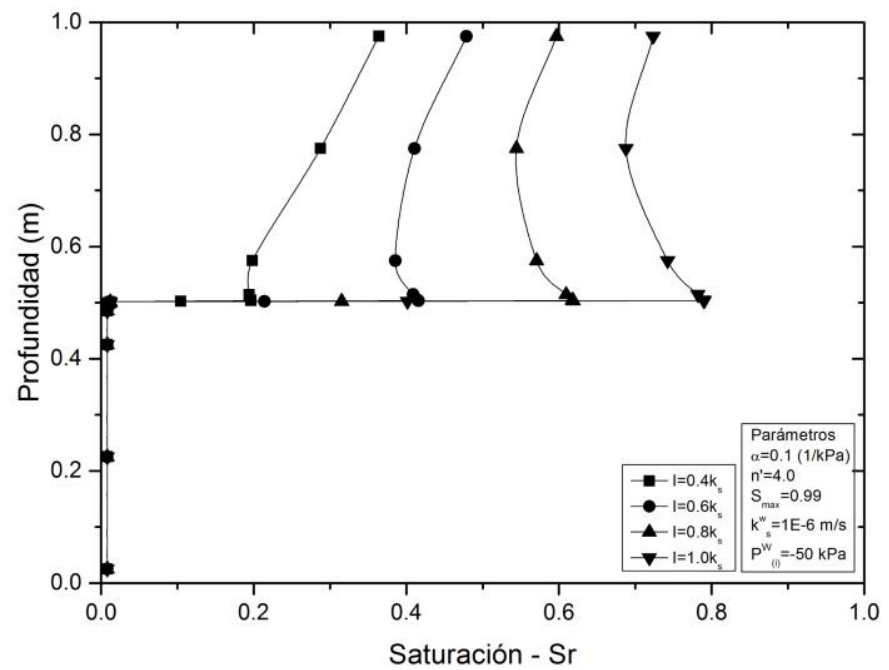

Fig. 8. Perfil de saturación para el modelo 2 sujeto a distintas intensidades $\left(\mathrm{I}=0.4,0.6,0.8\right.$ y $1.0 k^{W}$ ) en el tiempo $\mathrm{t}=45 \mathrm{~h}$.

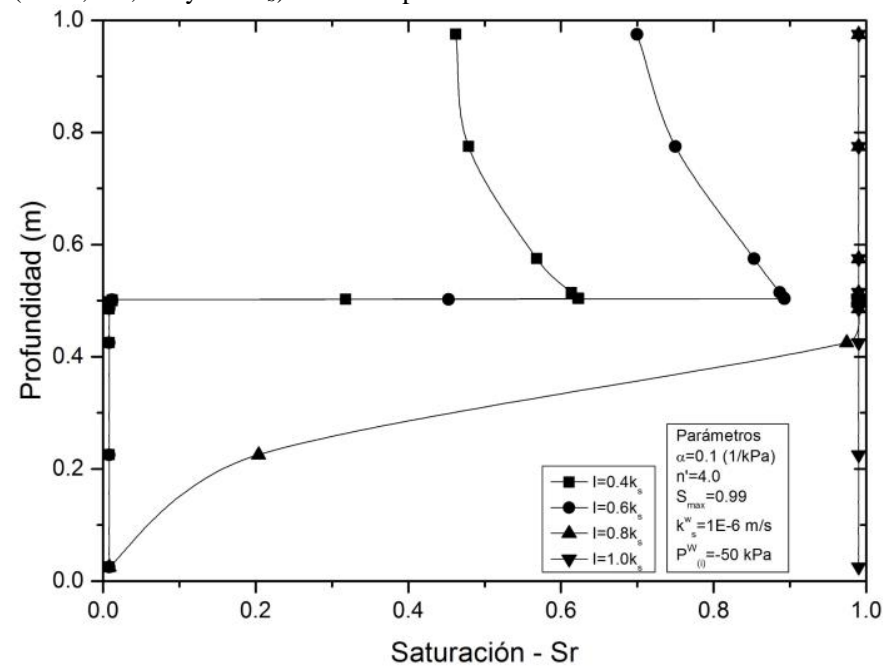

Fig. 9. Perfil de saturación para el modelo 2 sujeto a distintas intensidades (I=0.4, 0.6, 0.8 y $1.0 k^{W}$ ) en el tiempo $\mathrm{t}=80 \mathrm{~h}$.

Las Fig. 8 y 9 muestran que a mayor intensidad de lluvia mayor es el incremento de la presión de poros y por consiguiente mayor incremento de la saturación, resultado acorde con lo mencionado por Cai et al. [25]. Se presenta una influencia significativa de las características de la lluvia (Intensidad/duración) en el desarrollo de las presiones de poros en suelos parcialmente saturados. Es por esto que la relación II $k^{W}=1.0$ se puede catalogar como la condición más crítica para la estabilidad durante el proceso de infiltración, además se puede inferir que para las bajas intensidades no se alcanza a proporcionar el suficiente contenido de agua para superar la frontera hidráulica generada por la interacción suelogeosintético.

\section{E. Coeficiente de Permeabilidad saturada $\left(k^{W}\right)$}

Con el fin de analizar la influencia de las propiedades hidráulicas de los materiales en el rendimiento de la barrera capilar, en las Fig. 10 y 11 se muestran las variaciones de la presión de poros para permeabilidades de $k^{W}=1 \times 10^{-6}, 1.5 \times 10^{-6}$ y $2 \times 10^{-6} \mathrm{~m} / \mathrm{s}$, y sujetas a una lluvia constante de $\mathrm{I}=2.88 \mathrm{~mm} / \mathrm{h}$ y una presión de poros inicial de $-50 \mathrm{kPa}$.

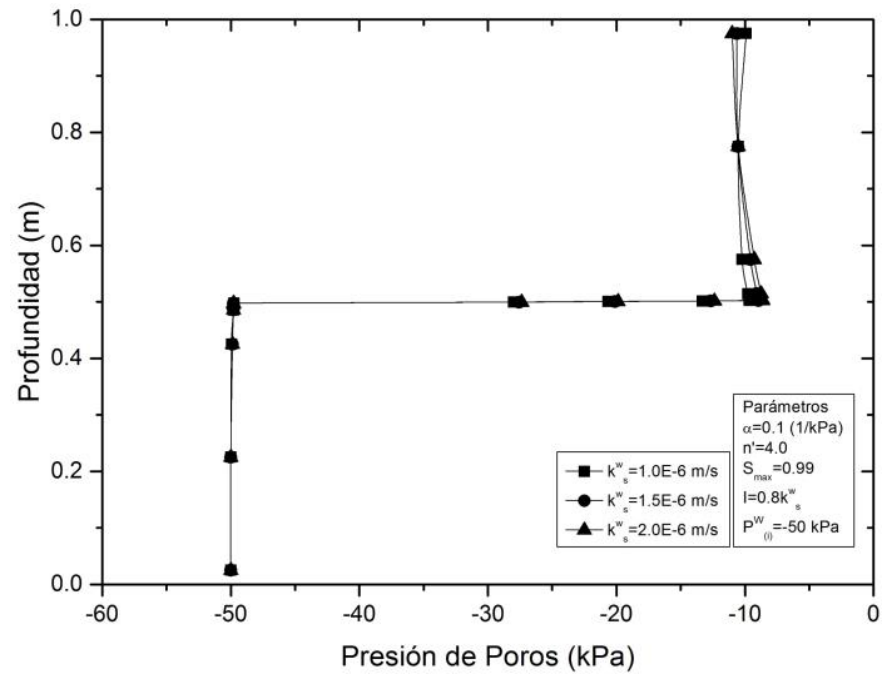

Fig. 10. Perfil de presión de poros para el modelo 2 bajo distintas permeabilidades $\left(k_{S}^{W}=1 \times 10^{-6}, 1.5 \times 10^{-6}\right.$ y $\left.2 \times 10^{-6} \mathrm{~m} / \mathrm{s}\right)$ en $\mathrm{t}=45 \mathrm{~h}$.

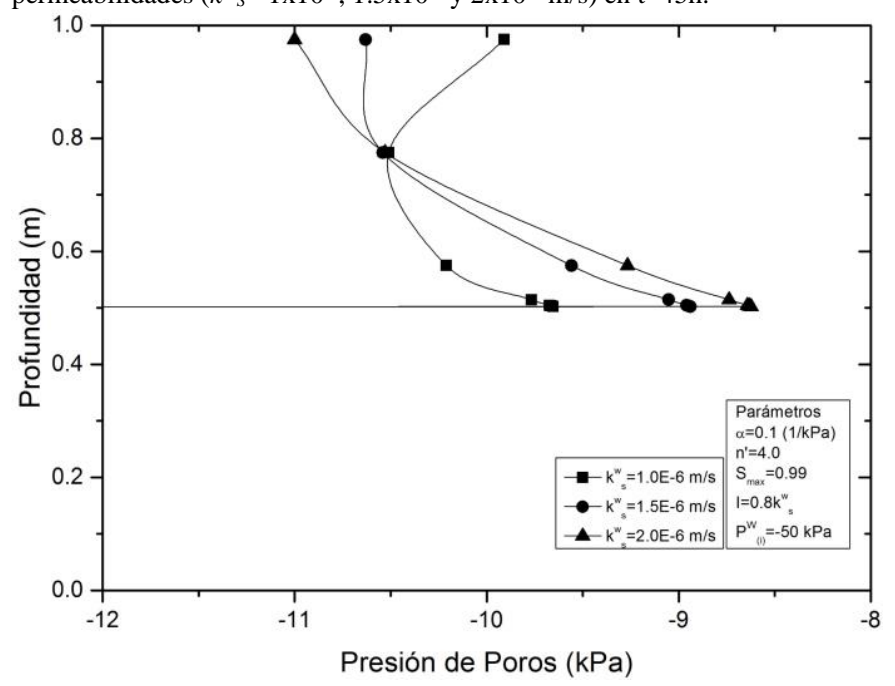

Fig. 11. Perfil de presión de poros para el modelo 2 en el intervalo $\mathrm{Pw}=-11 \mathrm{y}$ $8.5 \mathrm{kPa}$ bajo distintas permeabilidades $\left(k_{s}^{w}=\right.$ $1 \times 10^{-6}, 1.5 \times 10^{-6}$ y $\left.2 \times 10^{-6} \mathrm{~m} / \mathrm{s}\right)$ en $\mathrm{t}=45 \mathrm{~h}$.

La interrupción en el proceso de infiltración justamente en la mitad de la columna (posición del geosintético) se manifiesta para todas las permeabilidades como se indica en la Fig. 10, impidiendo el flujo de agua para los elementos de elevación menor a 0.5 metros y conservando la presión de poros inicial. Dicha concentración de agua permite resaltar que los valores más altos de presión de poros se presentaron en el caso de $k^{W}$ $=2 \times 10^{-6} \mathrm{~m} / \mathrm{s}$, alcanzando valores de $-8,6 \mathrm{kPa}$ en comparación con los obtenidos por $k^{W}=1 \times 10^{-6} \mathrm{~m} / \mathrm{s}$, los cuales fueron de $9,67 \mathrm{kPa}$ (ver Fig. 11). Este resultado es acorde con la facilidad de drenaje entre unidades de suelo que genera una mayor permeabilidad $\left(k^{W} S_{S}=2 \times 10^{-6} \mathrm{~m} / \mathrm{s}\right)$, permitiendo un proceso de infiltración más acelerado hasta llegar a las capas de geosintético.

Al mismo tiempo, se observa un mayor incremento de la presión de poros en los elementos más superficiales (ubicados en la parte superior de la columna) para el caso de $k^{W}=1 \times 10^{-6}$ $\mathrm{m} / \mathrm{s}$. Este resultado es consecuente con el análisis presentado por Cho et al. [26], puntualizando que para suelos con baja permeabilidad; el agua no drena de forma rápida, en efecto el aumento de la presión de poros se desarrolla cerca de la 
superficie; es decir, a medida que la permeabilidad disminuye el proceso de infiltración es más lento, generando que la acumulación de agua permanezca en los elementos más superficiales de la columna o el talud.

La Fig. 12 muestra el perfil de presión de poros para el tiempo de infiltración de 80 horas. De la Fig. 12 se observa que la variación de la permeabilidad no presenta mayor influencia en la ruptura de la barrera capilar, mostrando un comportamiento similar para todas las condiciones de permeabilidad planteadas.

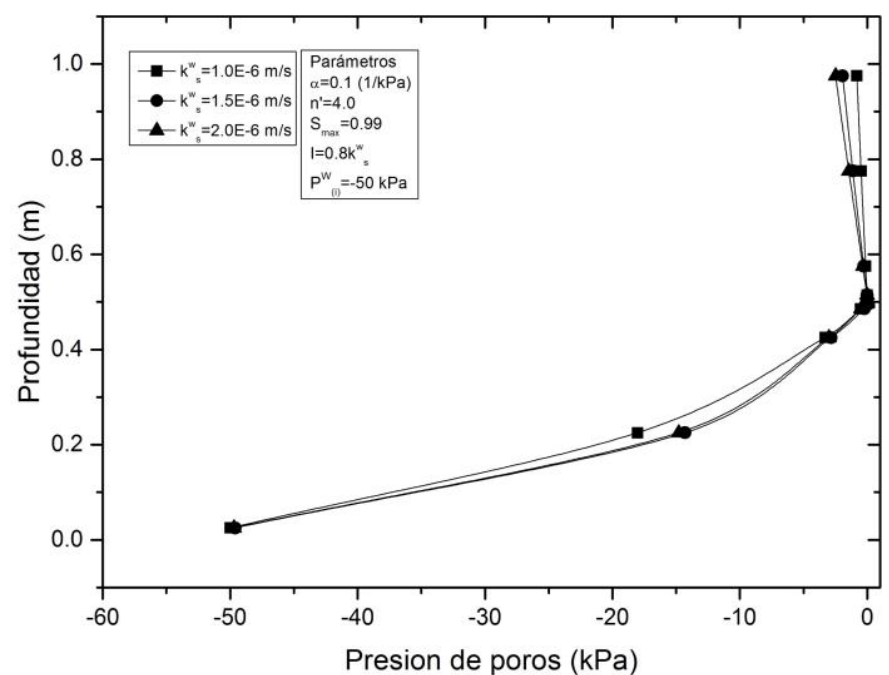

Fig. 12. Perfil de presión de poros para el modelo 2 bajo distintas permeabilidades $\left(k_{s}^{w}=1 \times 10^{-6}, 1.5 \times 10^{-6}\right.$ y $\left.2 \times 10^{-6} \mathrm{~m} / \mathrm{s}\right)$ en $\mathrm{t}=80 \mathrm{~h}$.

\section{CONCLUSIONES}

Mediante el modelo COMVI, se investigó la interacción del sistema suelo-geosintético bajo condiciones parcialmente saturadas y sujetas a lluvia de infiltración. A continuación, se presentan las principales conclusiones desarrolladas en el presente análisis unidimensional:

El uso del modelo arrojó resultados satisfactorios en relación con la capacidad e idoneidad de modelar de manera continua la interacción y el comportamiento hidráulico de varios materiales durante el proceso de infiltración. Es por esto que el modelo puede ser empleado para evaluar alternativas de solución en términos de reducir el efecto de la barrera capilar, propuestas tales como la inclusión de "cojines" de arena en la interface del suelo y el geosintético, presentada por Thuo et al.[13].

Mediante la variación espacio-temporal de la presión de poros y el grado de saturación se permite contrastar el proceso de infiltración a través de los modelos unidimensionales empleados. En el Modelo 1 se observa un flujo continuo y permanente a través de todos los elementos de la columna, el cual contrasta con el proceso interrumpido del modelo 2 debido a la interacción hidráulica del suelo y el geosintético.

La inclusión del geosintético en el Modelo 2, generó acumulación de agua en los elementos ubicados por encima de las capas de geosintético. Este modelo presenta un mayor grado de saturación en contraste con el Modelo 1, evidenciando la poca capacidad y/o efectividad del geosintético como material drenante bajo condiciones parciales de saturación. Por el contrario, el geotextil se convierte en una frontera impermeable dentro de la columna de suelo, interrumpiendo la disipación de presiones de poros durante y después de la lluvia de infiltración.

La concentración de agua por encima de las capas del geosintético permanece durante la mayoría del tiempo de infiltración y solamente cuando los elementos por encima de dichas capas alcanzan altos niveles de saturación cercanas al $100 \%$ y por consiguiente presiones de poros cercanas a cero, los elementos por debajo del geosintético experimentan de manera súbita acumulación de agua en sus poros. En efecto, grandes incrementos en presión de poros son necesarias para romper la barrera capilar impuesta por la interacción de materiales de distintas permeabilidades; es decir, el geosintético solo trabaja como material permeable en bajos rangos de succión.

Mediante el análisis paramétrico se permitió mostrar relaciones y variables que intervienen en la ocurrencia y rendimiento de la barrera capilar, resaltando la relación $I / k^{W}{ }_{S}$ como la de mayor influencia en el proceso de infiltración y en el desarrollo de la presión de poros. El efecto de la variable intensidad en el incremento de la presión de poros se desarrolla de forma directa, conforme al incremento de la intensidad los elementos por encima del geosintético presentan mayor contenido de agua, siendo el de mayor grado de saturación el escenario con $I=k_{S}^{W}$, conclusión acorde a resultados presentados en modelos bidimensionales [3], [11], [13], [14]. Resaltando el análisis unidimensional como una representación apropiada del proceso de infiltración en taludes y terraplenes.

Los resultados mediante la variación de la presión de poros inicial muestran una tendencia en el tiempo requerido para sobrepasar la barrera capilar, observándose que a menor presión de poros inicial; menor es el tiempo para superar las tensiones generadas en la interface entre el suelo y el geosintético. De esta manera que se puedan ocupar los vacíos del geosintético de agua y en consecuencia superar dicha barrera. El resultado plantea bases y preguntas para posteriores estudios del fenómeno en donde se analice la validez y comparación de dichos resultados con estudios experimentales.

En efecto, la implementación y diseño de estructuras de suelo con geosintético no tejido deben tener en cuenta el comportamiento de estos materiales bajo condiciones saturadas y parcialmente saturadas, junto con la presencia del fenómeno de barrera capilar en el proceso de infiltración y posteriores efectos en la capacidad de drenaje de taludes y terraplenes.

\section{REFERENCIAS}

[1] Y. Nakata, D. Liu, M. Hyodo, N. Yoshimoto, and Y. Kato, "Numerical simulation of an expressway embankment slope failure," Proc. Theor. Numer. Adv. Unsaturated Soil Mech., pp. $719-724,2010$

[2] J. G. Zornberg and J. K. Mitchell, "Reinforced Soil Structures with Poorly Draining Backfills Part II: Case Histories and Applications," Geosythetics Int., vol. 2, no. 1, pp. 265-307, 1995.

[3] T. Iryo and R. K. Rowe, "Infiltration into an embankment reinforced by nonwoven geotextiles," Can. Geotech. J., vol. 42, no. 4, pp. 1145-1159, 2005.

[4] B. C. Chattopadhyay and S. Chakravarty, "Application of jute geotextiles as facilitator in drainage," Geotext. Geomembranes, vol. 27, no. 2, pp. 156-161, 2009.

[5] S. A. Tan et al., "Large-scale drainage behaviour of composite geotextile and geogrid in residual soil," Geotext. Geomembranes, vol. 19, no. 3, pp. 163-176, 2001. 
[6] K. Terzaghi, Theoretical soil mechanics, vol. 18. Wiley Online Library, 1943.

[7] Bouazza, J. G. Zornberg, J. S. McCartney, and H. Nahlawi, "Significance of unsaturated behaviour of geotextiles in earthen structures," Aust. Geomech., vol. 41, no. 3, pp. 133-142, 2006.

[8] J. G. Zornberg, A. Bouazza, and J. S. McCartney, "Geosynthetic capillary barriers: current state of knowledge," Geosynth. Int., vol. 17, no. 5, pp. 273-300, 2010.

[9] I. R. Clough and W. J. French, "Laboratory and field work relating to the use of geotextiles in arid regions," in Proceedings of the 2nd International Conference on Geotextiles, Las Vegas, Nev, 1982, pp. $1-6$.

[10] G. N. Richardson, "Fundamental mistakes in slope design," Geotech. Fabr. Rep., vol. 15, no. 2, pp. 15-17, 1997.

[11] C. P. K. Gallage, T. Uchimura, and E. F. Garcia, "Function of permeable geosynthetics in unsaturated embankments subjected to rainfall infiltration," Geosynth. Int., vol. 14, no. 2, pp. 89-99, 2007.

[12] R. J. Bathurst, G. Siemens, and A. F. Ho, "Experimental investigation of infiltration ponding in one-dimensional sandgeotextile columns," Geosynth. Int., vol. 16, no. 3, pp. 158-172, 2009.

[13] J. N. Thuo, K. H. Yang, and C. C. Huang, "Infiltration into unsaturated reinforced slopes with nonwoven geotextile drains sandwiched in sand layers," Geosynth. Int., vol. 22, no. 6, pp. 457474, 2015.

[14] A. Jana, and A. Dey, "Effectiveness of Geotextile in Reinforced Soil Walls Backfilled With Effectiveness of Geotextile in Reinforced Soil Walls Backfilled," 2016, no. November, pp. 8-11.

[15] F. Oka, S. Kimoto, N. Takada, and Y. Higo, "A multiphase elastoviscoplastic analysis of an unsaturated river embankment associated with seepage flow," in Proceedings of the International Symposium on Prediction and Simulation Methods for Geohazard Mitigation. Kyoto, Japan, 2009, pp. 127-132.

[16] A. Jana and A. Dey, "EFFECTIVENESS OF GEOTEXTILE IN REINFORCED SOIL WALLS BACKFILLED WITH FINE SOIL," no. November, pp. 8-11, 2016.

[17] C. Jommi, "Remarks on the constitutive modelling of unsaturated soils," Exp. Evid. Theor. approaches unsaturated soils, pp. 139$153,2000$.

[18] M. T. Van Genuchten, "A closed-form equation for predicting the hydraulic conductivity of unsaturated soils," Soil Sci. Soc. Am. J., vol. 44, no. 5, pp. 892-898, 1980.

[19] L. M. Mendiola and L. Guadalupe, "Aplicación de modelos matemáticos para la obtención de la curva de retencion de humedad del suelo," Rev. Latinoam. Recur. Nat., 2010.

[20] E. Garcia, F. Oka, and S. Kimoto, "Numerical analysis of a onedimensional infiltration problem in unsaturated soil by a seepagedeformation coupled method," Int. J. Numer. Anal. Methods Geomech., vol. 35, no. 5, pp. 544-568, 2011.

[21] S. Kimoto, F. Oka, and E. García, "Numerical simulation of the rainfall infiltration on unsaturated soil slope considering a seepage flow," Geotech. Eng, vol. 44, no. 3, pp. 1-13, 2013.

[22] N. Lu and W. J. Likos, Unsaturated soil mechanics. Wiley, 2004.

[23] J. G. Zornberg, M. M. Azevedo, and C. B. Pickles, "Evaluation of the Development of Capillary Barriers at the Interface between Fine-Grained Soils and Nonwoven Geotextiles," Geotech. Spec. Publ., vol. 2016-Janua, no. 275 GSP, pp. 15-17, 2016.

[24] J. C. Stormont and C. E. Morris, "Method to estimate water storage capacity of capillary barriers," J. Geotech. Geoenvironmental Eng., vol. 124, no. 4, pp. 297-302, 1998.

[25] F. Cai and K. Ugai, "Numerical Analysis of Rainfall Effects on Slope Stability," no. June, pp. 69-78, 2004.

[26] S. E. Cho and S. R. Lee, "Instability of unsaturated soil slopes due to infiltration," Comput. Geotech., vol. 28, no. 3, pp. 185-208, 2001.
[27] J. Krahn, "Seepage modeling with SEEP/W: An engineering methodology," GEO-SLOPE Int. Ltd. Calgary, Alberta, Canada, 2004.

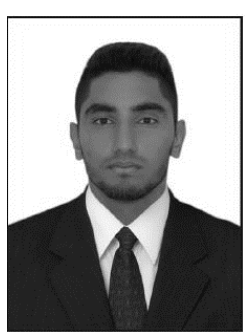

Juan Carlos Guzmán-Martínez nació en Itaguí, Antioquia, Colombia en 1994. En el 2017 se graduó de Ingeniería Civil en la Universidad de Antioquia, Colombia. Las áreas de interés investigativo incluyen la Geotecnia, estabilidad de taludes, infiltración en medios porosos, mecánica de suelos parcialmente saturados.

Durante el periodo de 2017-2018 participó en los estudios básicos de amenaza por movimientos en masa, inundaciones y avenidas torrenciales en los municipios de Caldas, La Estrella, Envigado, Itagüí, Bello, Copacabana y Barbosa del Área Metropolitana del Valle de Aburrá. Con énfasis en la determinación de la amenaza por movimientos en masa detonados por sismo y lluvia.

Actualmente se desempeña como estudiante de Maestría en Ingeniería con énfasis en Geotecnia y profesor de la Escuela Ambiental de la Facultad de Ingeniería de la Universidad de Antioquia.

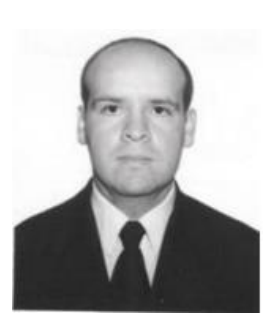

Edwin Fabián García Aristizábal nació en Pensilvania, Caldas, Colombia en 1977. Recibió su título de Ingeniero Civil de la Universidad Nacional de Colombia Sede Manizales en 1999, su grado de Master en Ingeniería de la Universidad de Tokyo, Japón en el año 2005 y su grado de Doctor en Ingeniería de la Universidad de Kyoto en el año 2010.

Actualmente se desempeña como profesor Asociado de la Escuela Ambiental de la Facultad de Ingeniería de la Universidad de Antioquia, Medellín, Colombia. Sus áreas de investigación incluyen los procesos de infiltración, suelos parcialmente saturados, estabilidad de taludes y análisis numérico.

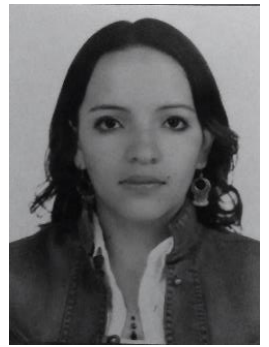

Alba Nury Gallego Hernández nació en Medellín, Antioquia, Colombia en 1978. Recibió su título de Ingeniera Geologa de la Universidad Nacional de Colombia Sede Medellín en 2003, sus grados de Master en Ciencias y Doctora en Ciencias de la Universidad de Shimane, Japón en los años 2006 y 2010, respectivamente.

Actualmente se desempeña como profesora Asistente de la Escuela Ambiental de la Facultad de Ingeniería de la Universidad de Antioquia, Medellín, Colombia. Sus áreas de investigación incluyen procesos geológicos de meteorización y erosión, petrografía de cementos y concretos y mineralogía aplicada. 
Carlos Alberto Vega-Posada nació en Bello, Antioquia, Colombia en 1979. Recibió su título de Ingeniero Civil de la Universidad Nacional de Colombia Sede Medellín en 2002, su grado de Master en Ingeniería de la Universidad de Ohio, Estados Unidos en el año 2008 y su grado de Doctor en Ingeniería de la Universidad de Northwestern en el año 2012.

Actualmente se desempeña como profesor Asociado de la Escuela Ambiental de la Facultad de Ingeniería de la Universidad de Antioquia, Medellín, Colombia. Sus áreas de investigación incluyen clasificación de suelos, problemas de interacción suelo-estructura, licuación de suelos, suelos parcialmente saturados e instrumentación y ensayos de campo y laboratorio. 